\title{
Structure of Hydrates of Metal Complexes with Barbituric Acids
}

\author{
Nicolay N. Golovnev*a, \\ Maxim S. Molokeev ${ }^{\mathrm{b}, \mathrm{a}}$ and Maxim K. Lesnikov ${ }^{\mathrm{a}}$ \\ ${ }^{a}$ Siberian Federal University \\ 79 Svobodny, Krasnoyarsk, 660041, Russia \\ ${ }^{b}$ Kirensky Institute of Physics, \\ Federal Research Center KSC SB RAS \\ 50/38 Akademgorodok, Krasnoyarsk, 660036, Russia
}

Received 12.03.2017, received in revised form 17.06.2017, accepted 20.08.2017

\begin{abstract}
Data on the synthesis and structure of metal complexes with barbituric $\left(\mathrm{H}_{2} \mathrm{ba}\right)$, thiobarbituric $\left(\mathrm{H}_{2} \mathrm{tba}\right)$ and 1,3-diethyl-2-thiobarbituric (HDetba) acids, which include a different number of coordinated water molecules, are summarized. Depending on the conditions, compounds of the composition $\left[\mathrm{M}\left(\mathrm{H}_{2} \mathrm{O}\right)_{n-k} L_{m}\right]^{0} \cdot \mathrm{pH}_{2} \mathrm{O}\left(\mathrm{M}^{m+}-\right.$ metal ion; $\mathrm{L}^{-}=\mathrm{Hba}^{-}, \mathrm{Htba} \mathrm{a}^{-}$u Detba $\left.; \mathrm{k} \geq 0, \mathrm{p} \geq 0\right)$ crystallize from the aqueous solution. The formation of neutral complexes of $\left[M\left(\mathrm{H}_{2} \mathrm{O}\right)_{n-k} \mathrm{~L}_{m}\right]^{0} \cdot \mathrm{pH}_{2} \mathrm{O}$ containing different amounts of coordinated water molecules $(n-k)$ occurs in the case of $L^{-}$ligands with potentially different dentateness and/or bridging coordination. Crystallization of several hydrates $\left[\mathrm{M}\left(\mathrm{H}_{2} \mathrm{O}\right)_{n-k} \mathrm{~L}_{m}\right]^{0} \cdot \mathrm{pH}_{2} \mathrm{O}$ is also expected if water molecules can be both terminal and bridged ligands in them, and central ions, for example, of alkaline earth metals, easily form different coordination polyhedra. Synthesis of new hydrates can significantly expand the range of available compounds, including potentially useful functional properties.
\end{abstract}

Keywords: barbituric acids, metal ions, complexes, hydrates, structure.

DOI: $10.17516 / 1998-2836-0036$.

(C) Siberian Federal University. All rights reserved

* Corresponding author E-mail address: ngolovnev@sfu-kras.ru 


\title{
Строение гидратов комплексов металлов с барбитуровыми кислотами
}

\author{
Н.Н. Головнев ${ }^{\mathrm{a}}$, М.С. Молокеев ${ }^{\boldsymbol{\sigma}, \mathrm{a}}$, М.К. Лесников ${ }^{\mathrm{a}}$ \\ ${ }^{a}$ Сибирский федеральный университет \\ Россия, 660041, Красноярск, пр. Свободный, 79 \\ ${ }^{6}$ Институт физики им. Л.В. Киренского, ФИЦ КНЦ СО РАН \\ Россия, 660036, Красноярск, Академгородок, 50/38
}

Обобщены данные о синтезе и структуре комплексов металлов с барбитуровой $\left(H_{2} b a\right)$, тиобарбитуровой (H $\left.H_{2} t b a\right)$ и 1,3-диэтил-2-тиобарбитуровой (HDetba) кислотами, в состав которых входит различное количество координированных молекул воды. В зависимости от условий из водного раствора кристаллизуются соединения состава $\left[\mathrm{M}\left(\mathrm{H}_{2} \mathrm{O}\right)_{n-k} L_{m}\right]^{0} \cdot \mathrm{pH}_{2} \mathrm{O}$ $\left(M^{m+}-\right.$ ион металла; $L^{-}=H b a^{-}, H t b a^{-}$и Detba $\left.; k \geq 0, p \geq 0\right)$. Образование нейтральных комплексов $\left[\mathrm{M}\left(\mathrm{H}_{2} \mathrm{O}\right)_{n-k} \mathrm{~L}_{m}\right]^{0} \cdot \mathrm{pH}_{2} \mathrm{O}$, содержащих разное количество координированных молекул воды $(n-k)$, имеет место в случае лигандов $L^{-}$с потенциально разной дентатностью и/или с мостиковым способом координации. Кристаллизаџия нескольких гидратов $\left.\left[\mathrm{M}_{(\mathrm{H}} \mathrm{O}\right)_{n-k} \mathrm{~L}_{m}\right]^{0} \cdot \mathrm{pH}_{2} \mathrm{O}$ также ожидается, если в них молекуль воды могут быть как концевыми, так и мостиковыми лигандами, а центральные ионы, например, щелочноземельных металлов, легко образуют разные координационные полиэдры. Синтез новых гидратов может существенно растирить круг доступных соединений, в том числе и с потенциально полезными функциональными свойствами.

Ключевые слова: барбитуровые кислоты, ионы металлов, комплексы, гидраты, структура.

В настоящее время наблюдается бурный рост числа синтезированных металлорганических комплексов, который во многом обязан их необычному строению и возможностью использования в качестве функциональных материалов [1]. Особый интерес вызывают полифункциональные лиганды, содержащие нейтральные и анионные связывающие центры. Такие лиганды могут образовывать с ионами металлов нейтральные комплексы, не содержащие дополнительные противоионы, которые могут заполнять потенциально полезные полости и каналы в структурах. К таким полифункциональным лигандам относится барбитуровая кислота $\left(\mathrm{H}_{2} \mathrm{ba}\right)$ и ее производные, например 2-тиобарбитуровая или тиобарбитуровая кислота $\left(\mathrm{H}_{2} \mathrm{tba}\right)$ и 1,3-диэтил-2-тиобарбитуровая кислота (HDetba) (рис. 1). Некоторые их производные используются в медицине и фармакологии [2], а их комплексы с металлами могут обладать полезной фармацевтической активностью [3].

Первая из этих кислот потенциально является N,N',O,O',O'”-донорным лигандом, а две другие - N,N',O,O',S-донорными лигандами. Наличие в барбитуровых кислотах расходящихся в пространстве центров связывания ионов металлов предполагает возможность образования ими координационных полимеров различного строения, в том числе и с полезными свойствами [4]. В настоящее время в основном определены структуры нейтральных комплексов металлов с 


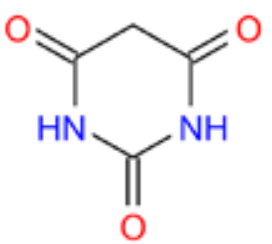

a)

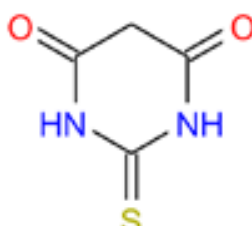

b)

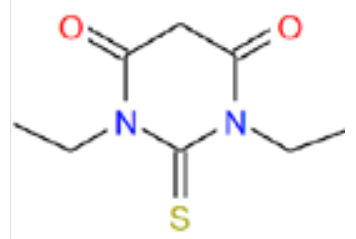

c)

Рис. 1.Графические формулы барбитуровых кислот: $a$-барбитуровая кислота $\left(\mathrm{H}_{2} \mathrm{ba}\right) ; b-2$-тиобарбитуровая кислота ( $\left.\mathrm{H}_{2} \mathrm{tba}\right) ; c-1,3$-диэтил-2-тиобарбитуровая кислота (HDetba)

Fig. 1. Graphic formulas of barbituric acids: $a$ - barbituric acid $\left(\mathrm{H}_{2} \mathrm{ba}\right) ; b-2$-thiobarbituric acid $\left(\mathrm{H}_{2}\right.$ tba); $c$-1,3-Diethyl-2-thiobarbituric acid (HDetba)

$\mathrm{H}_{2} \mathrm{ba}, \mathrm{H}_{2} \mathrm{tba}$ и их 5,5'-замещенными производными [4-6]. Для большинства из них охарактеризовано только по одной структуре, содержащей или не содержащей в своем составе воду. Как правило, для нейтральных комплексов с другими лигандами также описана только одна структура [6]. Однако недавно получены мономерные и полимерные комплексы ионов металлов $\left(\mathrm{M}^{m+}\right)$ с однозарядными анионами барбитуровых кислот, стехиометрический состав которых может быть формально представлен обобщенными формулами $\left[\mathrm{M}\left(\mathrm{H}_{2} \mathrm{O}\right)_{n} \mathrm{~L}_{\mathrm{m}}\right]^{0}$ или $\left[\mathrm{M}\left(\mathrm{H}_{2} \mathrm{O}\right)_{n-k} \mathrm{~L}_{m}\right]^{0} \cdot p \mathrm{H}_{2} \mathrm{O}$ $\left(\mathrm{M}^{m+}\right.$ - ион металла: $\mathrm{L}=\mathrm{Hba}^{-}, \mathrm{Htba}^{-}$и Detba $\left.; k \geq 0, p \geq 0\right)$. Последние содержат различное количество координированных молекул воды $(n-k)$ [7-13]. В настоящей работе сопоставлены структуры этих соединений, а также рассмотрены перспективы получения кристаллических нейтральных комплексов с разным количеством координированных молекул воды, основанные на учете только координирующих способностей центральных ионов, полифункциональных лигандов и молекул воды.

\section{Результаты и их обсуждение}

Вода играет большую роль в инженерии кристаллов комплексных соединений, в которых она может выступать в качестве концевых (терминальных) или мостиковых лигандов. Bce eе атомы в соединениях, кроме атома $\mathrm{O}$, связанного с ионом металла, могут участвовать в образовании различных межмолекулярных и внутримолекулярных водородных связей (BC), что влияет на упаковку кристаллической решетки и, следовательно, на физические и химические свойства. Например, кристаллизационная вода в молекулярных со-кристаллах может совершенно непредсказуемо влиять на их свойства, например термическую устойчивость [14]. В настоящей работе рассмотрены комплексы, в состав которых входят только два сорта лигандов: $\mathrm{H}_{2} \mathrm{O}$ и полифункциональный лиганд $\mathrm{L}^{-}$(иногда дополнительно $\mathrm{HL}^{0}$ ). Хорошо известен гидратный вид изомерии комплексов, проявляющийся в различном распределении одного и того же числа молекул воды и лиганда между внутренней координационной сферой и внешней сферой комплекса. Изменения формы координационного полиэдра или способа координации лиганда $\mathrm{L}^{-}$и/или молекул воды в нейтральном внутрисферном комплексе $\left[\mathrm{M}\left(\mathrm{H}_{2} \mathrm{O}\right)_{n} \mathrm{~L}_{\mathrm{m}}\right]$ также могут приводить к образованию нескольких его кристаллических изомеров $\left[\mathrm{M}\left(\mathrm{H}_{2} \mathrm{O}\right)_{n-k} \mathrm{~L}_{m}\right]^{0} k \mathrm{H}_{2} \mathrm{O}$ с разным количеством молекул кристаллизационной воды $(k)$. Кроме того, из водной среды могут осаждаться другие кристаллогидраты $\left[\mathrm{M}\left(\mathrm{H}_{2} \mathrm{O}\right)_{n-k} \mathrm{~L}_{m}\right]^{0} \cdot p \mathrm{H}_{2} \mathrm{O}(p \neq k)$ или соединения без молекул кристаллизационной воды.

$$
-403-
$$


Так, при различных условиях кристаллизации получены полимерные комплексы $\left[\mathrm{Na}_{2}\left(\mathrm{H}_{2} \mathrm{O}\right)_{x}(\mathrm{Htba})_{2}\right]\left(x=3,4\right.$ [7], 5 [8]). Монокристаллы соединения $\left[\mathrm{Na}_{2}\left(\mathrm{H}_{2} \mathrm{O}\right)_{3}\left(\mathrm{H}_{2} \mathrm{tba}\right)_{2}\right](\mathbf{1})$ выделяли из концентрированного горячего раствора при эквимолярном соотношении $\mathrm{H}_{2}$ tba и $\mathrm{NaOH}$. Комплекс $\left[\mathrm{Na}_{2}\left(\mathrm{H}_{2} \mathrm{O}\right)_{4}(\mathrm{Htba})_{2}\right](2)$ также получен кристаллизацией из такого же водного раствора, но при 2-3 ${ }^{\circ} \mathrm{C}$. Монокристаллы $\left[\mathrm{Na}_{2}\left(\mathrm{H}_{2} \mathrm{O}\right)_{5}(\mathrm{Htba})_{2}\right](3)$ образовывались при очень медленном испарении фильтрата. Структуры 1-3 показаны на рис. 2. Как следует из рис. 2 и таблицы, в октаэдрических комплексах реализуются разные способы координации тиобарбитурат-иона и молекул воды. Структуры 1 и 2 достаточно близки, структура 3 отличается от них большим числом концевых (немостиковых) молекул воды. Два независимых иона натрия в 2 или $\mathbf{3}$ имеют неэквивалентное лигандное окружение. Структура $\mathrm{Na}_{2}\left(\mathrm{H}_{2} \mathrm{O}\right)_{2} \mathrm{Cl}(\mathrm{Htba})$ [15] (рис. 2d) существенно отличается от структур 1-3, однако она содержит цепи -Na-H $\mathrm{H}_{2} \mathrm{O}-\mathrm{Na}-\mathrm{H}_{2} \mathrm{O}-\mathrm{Na}-. . .$, как и в 1 и 2. В ней мостиковыми являются все лиганды $\left(\mathrm{Htba}^{-}, \mathrm{Cl}^{-}\right.$и $\left.\mathrm{H}_{2} \mathrm{O}\right)$. Система $\mathrm{BC}$ во всех сравниваемых структурах также заметно отличается [7, 8, 15]. В гидратах 1 и 2 есть $\pi-\pi$-взаимодействие между ионами Htba- типа «голова-к-голове», в $3 \pi-\pi$-взаимодействие отсутствует.

Кристаллизацией из водного раствора выделены гидратный и безводный тиобарбитуратные комплексы калия. Солевой со-кристалл $\left[\mathrm{K}\left(\mathrm{H}_{2} \mathrm{O}\right)\left(\mathrm{H}_{2} \mathrm{tba}\right)(\mathrm{Htba})\right]_{n}$ получен при добавлении

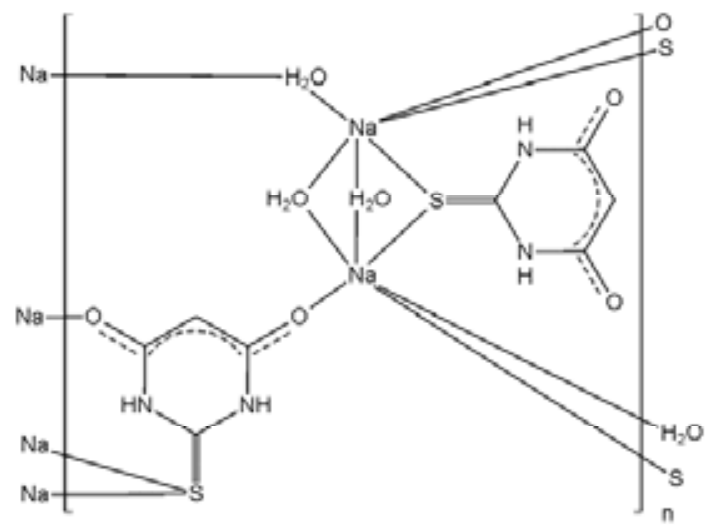

a)

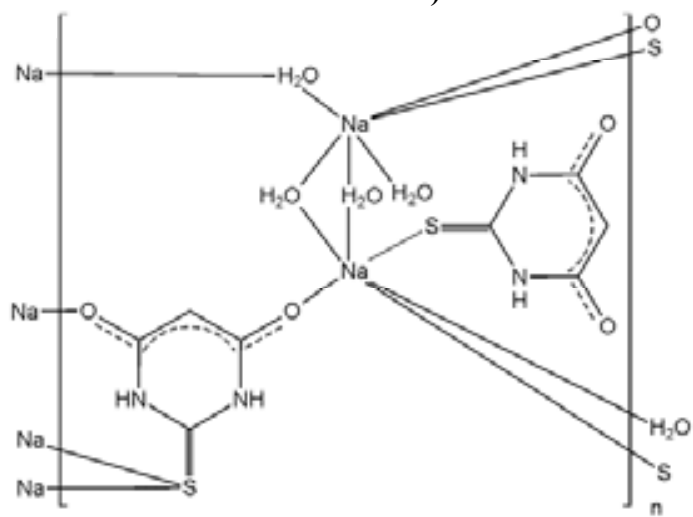

b)

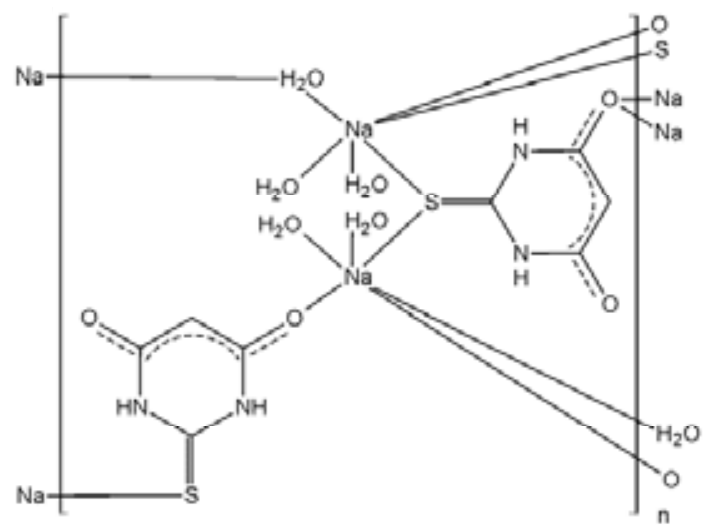

c)

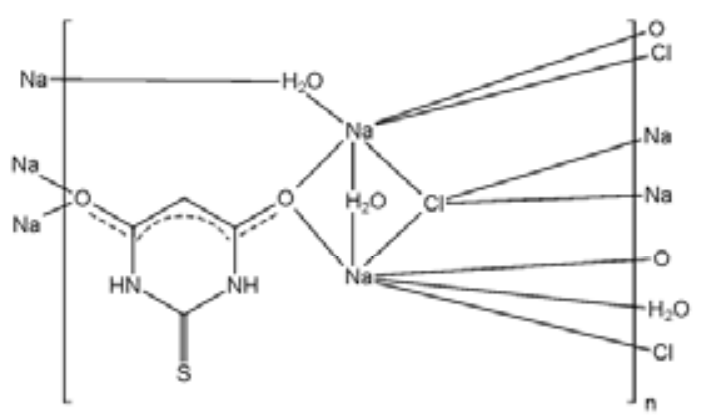

d)

Рис. 2. Строение $\left.\left.\left.\left[\mathrm{Na}_{2}\left(\mathrm{H}_{2} \mathrm{O}\right)_{x}(\mathrm{Htba})_{2}\right]: a\right)-x=3(1) ; b\right)-x=4(2) ; c\right)-x=5$ и $\left.d\right) \mathrm{Na}_{2} \mathrm{Cl}(\mathrm{Htba})\left(\mathrm{H}_{2} \mathrm{O}\right)_{2}$

Fig. 2. Structure of $\left.\left.\left.\left[\mathrm{Na}_{2}\left(\mathrm{H}_{2} \mathrm{O}\right)_{x}(\mathrm{Htba})_{2}\right]: a\right)-x=3(\mathbf{1}) ; b\right)-x=4(2) ; c\right)-x=5$ и $\left.d\right) \mathrm{Na}_{2} \mathrm{Cl}(\mathrm{Htba})\left(\mathrm{H}_{2} \mathrm{O}\right)_{2}$ 
Таблица 1. Данные о структуре гидратов барбитуратных комплексов металлов

Table 1. The structure of hydrated barbiturate metal complexes

\begin{tabular}{|c|c|c|c|c|}
\hline Комплекс & $\begin{array}{c}\text { Форма } \\
\text { полиэдра }\end{array}$ & $\begin{array}{c}\text { Тип координации } \\
\text { барбитуратного } \\
\text { лиганда }\end{array}$ & $\begin{array}{c}\text { Тип координации } \\
\text { молекул воды }\end{array}$ & Ссылка \\
\hline$\left[\mathrm{Na}_{2}\left(\mathrm{H}_{2} \mathrm{O}\right)_{3}(\mathrm{Htba})_{2}\right]_{n}(\mathbf{1})$ & октаэдр & $\begin{array}{l}\mu_{4}-\mathrm{O}, \mathrm{O}^{\prime}, \mathrm{S}, \mathrm{S} ; \\
\mu_{2}-\mathrm{S}, \mathrm{S}\end{array}$ & $\mu_{2}-\mathrm{H}_{2} \mathrm{O}$ & \multirow{3}{*}[7,8]{} \\
\hline$\left[\mathrm{Na}_{2}\left(\mathrm{H}_{2} \mathrm{O}\right)_{4}(\mathrm{Htba})_{2}\right]_{n}(\mathbf{2})$ & октаэдр & $\begin{array}{l}\mu_{4}-\mathrm{O}, \mathrm{O}, \mathrm{S}, \mathrm{S} ; \\
\text { терминальный-O }\end{array}$ & $\begin{array}{l}\mu_{2}-\mathrm{H}_{2} \mathrm{O} ; \\
\text { терминальный }\end{array}$ & \\
\hline$\left[\mathrm{Na}_{2}\left(\mathrm{H}_{2} \mathrm{O}\right)_{5}(\mathrm{Htba})_{2}\right]_{n}(\mathbf{3})$ & октаэдр & $\begin{array}{l}\mu_{4}-\mathrm{O}, \mathrm{O}, \mathrm{S}, \mathrm{S} ; \\
\mu_{2}-\mathrm{O}, \mathrm{S}\end{array}$ & $\begin{array}{l}\mu_{2}-\mathrm{H}_{2} \mathrm{O} ; \\
\text { терминальный }\end{array}$ & \\
\hline$\left[\mathrm{Ca}\left(\mathrm{H}_{2} \mathrm{O}\right)_{5}(\mathrm{Htba})_{2}\right] \cdot 2 \mathrm{H}_{2} \mathrm{O}(4)$ & \multirow{2}{*}{$\begin{array}{l}\text { одношапочная } \\
\text { тригональная призма }\end{array}$} & терминальный-О & терминальный & \multirow{3}{*}{ [9] } \\
\hline$\left[\mathrm{Ca}_{2}\left(\mathrm{H}_{2} \mathrm{O}\right)_{8}(\mathrm{Htba})_{4}\right](\mathbf{5})$ & & $\begin{array}{l}\mu_{2}-\mathrm{O}, \mathrm{O} ; \\
\text { терминальный-О }\end{array}$ & терминальный & \\
\hline$\left[\mathrm{Sr}\left(\mathrm{H}_{2} \mathrm{O}\right)_{4}(\mathrm{Htba})_{2}\right]_{n}(\mathbf{6})$ & квадратная антипризма & $\mu_{2}-\mathrm{O}, \mathrm{S}$ & терминальный & \\
\hline$\left[\mathrm{Sr}\left(\mathrm{H}_{2} \mathrm{O}\right)_{4}(\mathrm{Htba})_{2}\right]_{n} \cdot n \mathrm{H}_{2} \mathrm{O}(7)$ & $\begin{array}{l}\text { трехшапочная } \\
\text { тригональная призма }\end{array}$ & $\begin{array}{l}\mu_{2}-\mathrm{O}, \mathrm{O} ; \\
\text { терминальный-О }\end{array}$ & $\begin{array}{l}\mu_{2}-\mathrm{H}_{2} \mathrm{O} ; \\
\text { терминальный }\end{array}$ & {$[9,10]$} \\
\hline$\left[\mathrm{Ba}\left(\mathrm{H}_{2} \mathrm{O}\right)_{2}(\mathrm{Htba})_{2}\right]_{n}(\mathbf{8})$ & квадратная антипризма & $\mu_{3}-\mathrm{O}, \mathrm{S}, \mathrm{S}$ & терминальный & \multirow[b]{2}{*}[11]{} \\
\hline$\left[\mathrm{Ba}\left(\mathrm{H}_{2} \mathrm{O}\right)_{3}(\mathrm{Htba})_{2}\right]_{n} \cdot 2 n \mathrm{H}_{2} \mathrm{O}(\mathbf{9})$ & $\begin{array}{l}\text { трехшапочная } \\
\text { тригональная призма }\end{array}$ & $\begin{array}{l}\mu_{4}-\mathrm{O}, \mathrm{O}, \mathrm{O}, \mathrm{S} ; \\
\text { терминальный-O }\end{array}$ & $\begin{array}{l}\mu_{2}-\mathrm{H}_{2} \mathrm{O} ; \\
\text { терминальный }\end{array}$ & \\
\hline$\left[\mathrm{Co}\left(\mathrm{H}_{2} \mathrm{O}\right)_{2}(\mathrm{Hba})_{2}\right]_{n}(\mathbf{1 0})$ & октаэдр & $\mu_{2}-\mathrm{O}, \mathrm{O}$ & терминальный & \multirow{2}{*}[12]{} \\
\hline$\left[\mathrm{Co}\left(\mathrm{H}_{2} \mathrm{O}\right)_{4}(\mathrm{Hba})_{2}\right](\mathbf{1 1})$ & октаэдр & терминальный-О & терминальный & \\
\hline$\left[\mathrm{Mn}\left(\mathrm{H}_{2} \mathrm{O}\right)_{2}(\mathrm{Detba})_{2}\right]_{n}(\mathbf{1 2})$ & октаэдр & $\mu_{2}-\mathrm{O}, \mathrm{O}^{\prime}$ & терминальный & \multirow{3}{*}{ [13] } \\
\hline$\left[\mathrm{Mn}_{3}\left(\mathrm{H}_{2} \mathrm{O}\right)_{10}(\text { Detba })_{6}\right](\mathbf{1 3})$ & октаэдр & $\begin{array}{l}\mu_{2}-\mathrm{O}, \mathrm{O} ; \\
\text { терминальный-О }\end{array}$ & терминальный & \\
\hline$\left[\mathrm{Mn}\left(\mathrm{H}_{2} \mathrm{O}\right)_{4}(\mathrm{Detba})_{2}\right] \cdot \mathrm{H}_{2} \mathrm{O}(\mathbf{1 4})$ & октаэдр & терминальный-О & терминальный & \\
\hline
\end{tabular}

к нейтрализованному КОН водному раствору $\mathrm{H}_{2}$ tba метанольного раствора $\left(n-\mathrm{C}_{4} \mathrm{H}_{9}\right)_{2} \mathrm{SnCl}_{2}[16]$ или метанольного раствора $\mathrm{Ph}_{3} \mathrm{SnCl}$ [17]. Безводный тиобарбитурат калия кристаллизуется непосредственно из водного раствора [16].

В $\left[\mathrm{K}\left(\mathrm{H}_{2} \mathrm{O}\right)\left(\mathrm{H}_{2} \mathrm{tba}\right)(\mathrm{Htba})\right]_{n}$ к иону $\mathrm{K}^{+}$координирована одна молекула воды, а также через атомы $\mathrm{S}$ или $\mathrm{O}$ четыре иона $\mathrm{Htba}^{-}$и три молекулы $\mathrm{H}_{2}$ tba. При этом по одному атому кислорода в $\mathrm{Htba}^{-}$и $\mathrm{H}_{2}$ tba остаются не связанными с ионом $\mathrm{K}^{+}$(рис. $3 a$ ). В результате получается координационный полиэдр в форме искаженной квадратной антипризмы. Каждые два полиэдра соединяются между собой четырехугольными гранями из атомов $\mathrm{S}$ в пары, которые ребрами, так же через атомы $\mathrm{S}$, объединяются в бесконечные цепочки. Под некоторым углом к этим цепочкам находятся параллельные слои из молекул и анионов 2-тиобарбитуровой кислоты. Структура стабилизирована ВС и $\pi-\pi$-взаимодействием между $\mathrm{Htba}^{-}$и $\mathrm{H}_{2} \mathrm{tba}$. Координация нейтральных лигандов, в том числе и совместно с координацией сопряженных с ними анионов, как в сокристаллах $\mathrm{M}\left(\mathrm{H}_{2} \mathrm{ba}\right)(\mathrm{Hba}) \cdot 2 \mathrm{H}_{2} \mathrm{O}(\mathrm{M}=\mathrm{Na}, \mathrm{K}), \mathrm{K}\left(\mathrm{H}_{2} \mathrm{ba}\right)_{0.5}(\mathrm{Hba}) \cdot 1.5 \mathrm{H}_{2} \mathrm{O}$ [18] и $\mathrm{Rb}\left(\mathrm{H}_{2} \mathrm{ba}\right)(\mathrm{Hba}) \cdot \mathrm{H}_{2} \mathrm{O}$ [19], расширяет список гидратов, которые можно выделить из водного раствора.

В безводном соединении (рис. $3 b$ ) каждый ион калия связан с четырьмя атомами О и двумя атомами $\mathrm{S}$ ионов $\mathrm{Htba}^{-}$с образованием искаженного октаэдра, причем $\mathrm{S}$-координированные лиганды $\mathrm{Htba}^{-}$находятся в транс-положении друг к другу. Октаэдры соединяются между собой

$$
-405-
$$




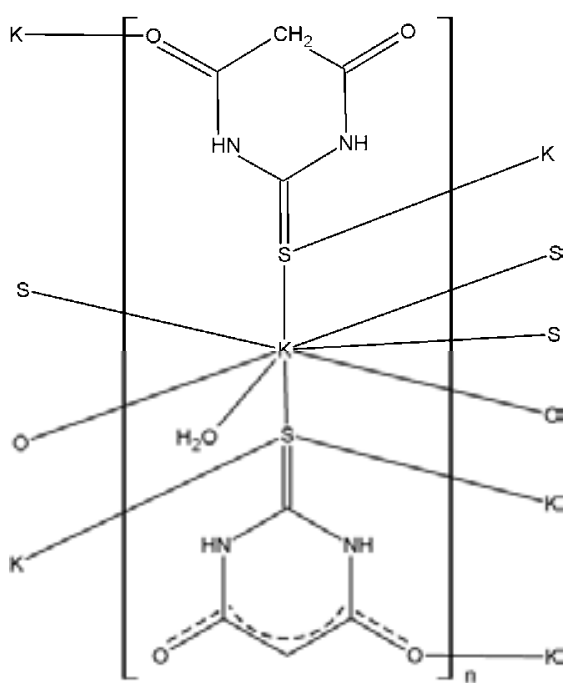

a)

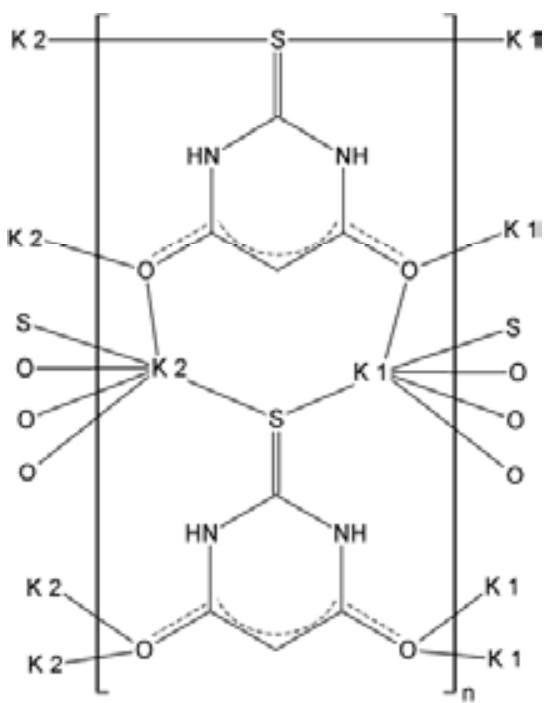

b)

Рис. 3. Химические связи в $\left[\mathrm{K}\left(\mu_{4}-\mathrm{Htba}-\mathrm{O}, \mathrm{S}, \mathrm{S}, \mathrm{S}\right)\left(\mu_{3}-\mathrm{H}_{2} \mathrm{tba}-\mathrm{O}, \mathrm{S}, \mathrm{S}\right)\left(\mathrm{H}_{2} \mathrm{O}\right)\right]_{n}(a)$ и $\left[\mathrm{K}\left(\mu_{6}-\mathrm{Htba}-\mathrm{O}, \mathrm{O}, \mathrm{O}^{\prime}, \mathrm{O}^{\prime}, \mathrm{S}, \mathrm{S}\right)\right]_{n}(b)$

Fig. 3. Chemical bonds in $\left[\mathrm{K}\left(\mu_{4}-\mathrm{Htba}-\mathrm{O}, \mathrm{S}, \mathrm{S}, \mathrm{S}\right)\left(\mu_{3}-\mathrm{H}_{2} \mathrm{tba}-\mathrm{O}, \mathrm{S}, \mathrm{S}\right)\left(\mathrm{H}_{2} \mathrm{O}\right)\right]_{n}(a)$ и $\left[\mathrm{K}\left(\mu_{6}-\mathrm{Htba}-\mathrm{O}, \mathrm{O}, \mathrm{O}^{\prime}, \mathrm{O}^{\prime}, \mathrm{S}, \mathrm{S}\right)\right]_{n}(b)$

вершинами и ребрами в бесконечный слой. С помощью ВC $\mathrm{N}-\mathrm{H} \cdots \mathrm{O}$ и $\mathrm{C}-\mathrm{H} \cdots \mathrm{S}$ слои из октаэдров объединяются в трехмерный каркас. Структура также стабилизирована $\pi-\pi$-взаимодействием между $\mathrm{Htba}^{-}$типа «голова-к-голове».

Комплексы $\left[\mathrm{Ca}\left(\mathrm{H}_{2} \mathrm{O}\right)_{5}(\mathrm{Htba})_{2}\right] \cdot 2 \mathrm{H}_{2} \mathrm{O}$ и $\left[\mathrm{Ca}_{2}\left(\mathrm{H}_{2} \mathrm{O}\right)_{8}(\mathrm{Htba})_{4}\right]$ получены нейтрализацией 2-тиобарбитуровой кислоты карбонатом кальция [9]. Кристаллизацией из водного раствора при $60{ }^{\circ} \mathrm{C}$ выделен $\left[\mathrm{Ca}\left(\mathrm{H}_{2} \mathrm{O}\right)_{5}(\mathrm{Htba})_{2}\right] \cdot 2 \mathrm{H}_{2} \mathrm{O}$, а при $20^{\circ} \mathrm{C}-\left[\mathrm{Ca}_{2}\left(\mathrm{H}_{2} \mathrm{O}\right)_{8}(\mathrm{Htba})_{4}\right]$.

Ион $\mathrm{Ca}^{2+}$ в $\left[\mathrm{Ca}\left(\mathrm{H}_{2} \mathrm{O}\right)_{5}(\mathrm{Htba})_{2}\right] \cdot 2 \mathrm{H}_{2} \mathrm{O}(4)$ окружен семью атомами кислорода от пяти молекул воды и двух концевых ионов $\mathrm{Htba}^{-}$с образованием одношапочной тригональной призмы (рис. 4a). Полиэдры $\mathrm{CaO}_{7}$ не имеют общих вершин, ребер и граней, а связываются лишь за счет ВC, т.е. структура комплекса является островной. В $\left[\mathrm{Ca}_{2}\left(\mathrm{H}_{2} \mathrm{O}\right)_{8}(\mathrm{Htba})_{4}\right](\mathbf{5})$ каждый ион $\mathrm{Ca}^{2+}$ связан с семью атомами кислорода от четырех молекул воды, одного терминального иона $\mathrm{Htba}^{-}$и двух мостиковых $\mathrm{Htba}^{-}$(рис. 4b), формируя одношапочную тригональную призму. Мостиковые лиганды связывают призмы в пары с образованием островной структуры, состоящей из биядерных комплексов. За счет водородных связей эти пары призм образуют протяженные цепи. Два лиганда $\mu_{2}-\mathrm{Htba}^{-}$соединяются с двумя катионами $\mathrm{Ca}^{2+}$ и замыкают 12-членный цикл, такие же циклы встречаются и в комплексах других металлов с органическими лигандами [4]. Многочисленные межмолекулярные ВC O-H $\cdots \mathrm{O}, \mathrm{N}-\mathrm{H} \cdots \mathrm{O}, \mathrm{N}-\mathrm{H} \cdots \mathrm{S}$ и O-H ‥S генерируют в 5 и 4 трехмерную сеть. Дополнительно эти структуры стабилизируют $\pi-\pi$-взаимодействия между гетероциклическими ионами $\mathrm{Htba}^{-}$, которые упакованы по типу «голова-к-хвосту».

Нейтрализацией $\mathrm{SrCO}_{3} 2$-тиобарбитуровой кислотой в воде при $60{ }^{\circ} \mathrm{C}$ получен кристаллогидрат $\left[\mathrm{Sr}\left(\mathrm{H}_{2} \mathrm{O}\right)_{4}(\mathrm{Htba})_{2}\right]_{n} \cdot n \mathrm{H}_{2} \mathrm{O}[10]$. Комплекс $\left[\mathrm{Sr}\left(\mathrm{H}_{2} \mathrm{O}\right)_{4}(\mathrm{Htba})_{2}\right]_{\mathrm{n}}$ накапливается в небольшом ко- 


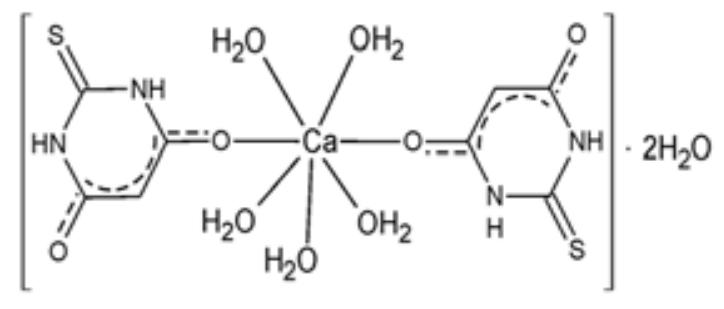

a)

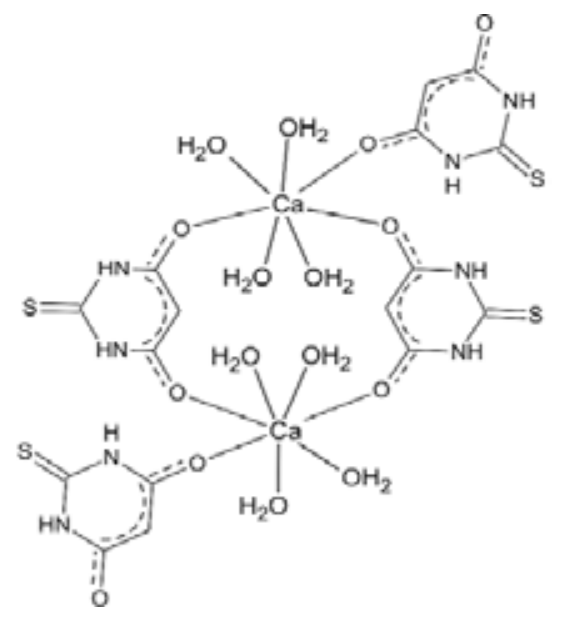

b)

Рис. 4. Строение комплексов $4(a)$ и $5(b)$

Fig. 4. Structure of complexes $\mathbf{4}(a)$ and $\mathbf{5}(b)$

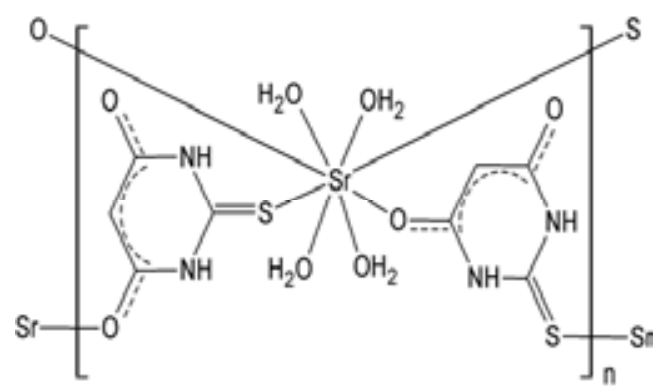

a)

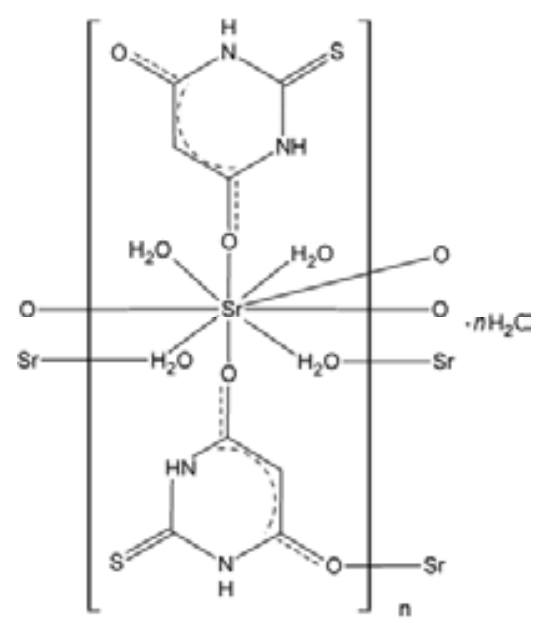

b)

Рис. 5. Химические связи в комплексах $6(a)$ и $7(b)$

Fig. 5. Chemical bonds in complexes $6(a)$ and $7(b)$

личестве только при продолжительном испарении полученного в предыдущем синтезе фильтрата в течение $\sim 30$ дней [9].

Катион $\mathrm{Sr}^{2+}$ в $\left[\mathrm{Sr}\left(\mathrm{H}_{2} \mathrm{O}\right)_{4}(\mathrm{Htba})_{2}\right]_{\mathrm{n}}(\mathbf{6})$ имеет координационное число, равное восьми. К нему координированы два иона $\mathrm{Htba}^{-}$через атомы кислорода, два Htba- через атомы серы и четыре молекулы $\mathrm{H}_{2} \mathrm{O}$ (рис. 5a), образуя искаженную квадратную антипризму. Полиэдры $\mathrm{SrO}_{6} \mathrm{~S}_{2} \mathrm{He}$ соприкасаются друг с другом непосредственно, а связаны мостиковыми лигандами Htba- с образованием бесконечного слоя. Каждая квадратная антипризма связана с тремя такими же с помощью двух независимых лигандов $\mu_{2}-\mathrm{O}, \mathrm{S}-\mathrm{Htba}^{-}$. Причем с каждой из двух антипризм она

$$
-407 \text { - }
$$


связана только одним мостиковым лигандом $\mathrm{Htba}^{-}$, но с разной его координацией к двум центральным ионам: либо через $\mathrm{O}$, либо через $\mathrm{S}$. C третьей антипризмой ее соединяют сразу два мостиковых лиганда с координацией к $\operatorname{Sr}(\mathrm{II})$ одного лиганда через атом $\mathrm{O}$, а другого через атом $\mathrm{S}$, что приводит к замыканию 12-членного цикла, как в случае комплекса $\left[\mathrm{Ca}_{2}\left(\mathrm{H}_{2} \mathrm{O}\right)_{8}(\mathrm{Htba})_{4}\right]$

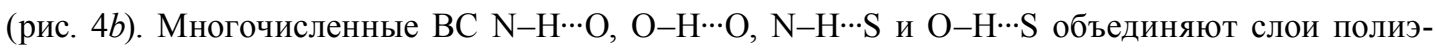
дров в трехмерный каркас. Структура стабилизирована $\pi-\pi$-взаимодействием типа «голова-кголове».

$\mathrm{B}\left[\mathrm{Sr}\left(\mathrm{H}_{2} \mathrm{O}\right)_{4}(\mathrm{Htba})_{2}\right]_{n} \cdot n \mathrm{H}_{2} \mathrm{O}(7)$ ион $\mathrm{Sr}^{2+}$ окружен девятью атомами кислорода (рис. $\left.5 b\right)$ с образованием почти идеальной трехшапочной тригональной призмы. При этом к $\mathrm{Sr}^{2+}$ монодентатно координированы три иона $\mathrm{Htba}^{-}$и шесть молекул воды (четыре $\mu_{2}$-мостиковые и две концевые). Одна молекула воды является кристаллизационной. Три атома кислорода координированных ионов Htba- составляют одну из граней полиэдра. Трехшапочные тригональные призмы соединяются друг с другом треугольными гранями, которые лежат в их основании, и каждая из них образована одним атомом кислорода иона $\mathrm{Htba}^{-}$и двумя атомами кислорода мостиковых молекул воды. При этом образуются бесконечные цепи, в которых можно выделить 4-членные циклы. ВC $\mathrm{N}-\mathrm{H} \cdots \mathrm{O}$ и $\mathrm{N}-\mathrm{H} \cdots \mathrm{S}$ связывают между собой цепочки из полиэдров в бесконечные слои. В структуре отсутствует $\pi-\pi$-взаимодействие.

Реакций между эквимолярными количествами $\mathrm{BaCO}_{3}$ и $\mathrm{H}_{2} \mathrm{tba}$ в воде при $60{ }^{\circ} \mathrm{C}$ получены кристаллы $\left[\mathrm{Ba}\left(\mathrm{H}_{2} \mathrm{O}\right)_{2}(\mathrm{Htba})_{2}\right]_{n}$, а при медленном испарении полученного фильтрата выделены монокристаллы $\left[\mathrm{Ba}\left(\mathrm{H}_{2} \mathrm{O}\right)_{3}(\mathrm{Htba})_{2}\right]_{n} \cdot 2 n \mathrm{H}_{2} \mathrm{O}[11]$.

$\mathrm{B}\left[\mathrm{Ba}\left(\mathrm{H}_{2} \mathrm{O}\right)_{2}(\mathrm{Htba})_{2}\right]_{n}(\mathbf{8})$ ион $\mathrm{Ba}^{2+}$ окружен четырьмя атомами серы и четырьмя атомами кислорода. Он связан с шестью ионами $\mu_{3}-\mathrm{Htba}-\mathrm{O}, \mathrm{S}, \mathrm{S}$ (четыре связаны через атомы $\mathrm{S}$ и два через атомы О) и двумя концевыми молекулами воды (рис. $6 a$ ) с образованием искаженной квадратной антипризмы. Квадратные антипризмы объединены ребрами посредством мостиковых атомов серы от двух лигандов в бесконечные цепи, при этом замыкаются 4- и 12-членные циклы. Каждый ион $\mathrm{Ba}^{2+}$ связан лигандами $\mu_{3}-\mathrm{Htba}-\mathrm{O}, \mathrm{S}, \mathrm{S}$ с двумя другими ионами $\mathrm{Ba}^{2+}$ каждой из соседних цепей с образованием трехмерной структуры. ВС $\mathrm{N}-\mathrm{H} \cdots \mathrm{O}$ и $\pi-\pi$-взаимодействие типа «голова-к-голове» стабилизируют структуру.

Ион $\mathrm{Ba}^{2+}$ в $\left[\mathrm{Ba}\left(\mathrm{H}_{2} \mathrm{O}\right)_{3}(\mathrm{Htba})_{2}\right]_{n} 2 n \mathrm{H}_{2} \mathrm{O}$ (9) окружают восемь атомов кислорода и один атом серы с образованием трехшапочной тригональной призмы. Он связан с четырьмя молекулами воды (две терминальные и две $\mu_{2}$-мостиковые) и пятью ионами $\mathrm{Htba}^{-}$(рис. $6 b$ ). Два полиэдра $\mathrm{BaO}_{8} \mathrm{~S}$ имеют общую четырехугольную грань, состоящую из двух атомов О от двух лигандов $\mu_{4}-\mathrm{Htba}-\mathrm{O}, \mathrm{O}, \mathrm{O}$ ', $\mathrm{S}$ и двух атомов $\mathrm{O}$ от двух $\mu_{2}-\mathrm{H}_{2} \mathrm{O}$. Каждый полиэдр связан четырьмя мостиковыми ионами $\mathrm{Htba}^{-}$с семью такими же полиэдрами с образованием слоев. В структуре есть два типа 12-членных циклов, первый из них присутствует в $\left[\mathrm{Ca}_{2}\left(\mathrm{H}_{2} \mathrm{O}\right)_{8}(\mathrm{Htba})_{4}\right]$, а второй - в $\left[\mathrm{Ba}\left(\mathrm{H}_{2} \mathrm{O}\right)_{2}(\mathrm{Htba})_{2}\right]_{n}$. Межмолекулярные $\mathrm{BC} \mathrm{N}-\mathrm{H} \cdots \mathrm{O}, \mathrm{N}-\mathrm{H} \cdots \mathrm{S}, \mathrm{O}-\mathrm{H} \cdots \mathrm{O}$ и $\mathrm{O}-\mathrm{H} \cdots \mathrm{S}$ образуют в 9 трехмерный каркас, а $\pi-\pi$-взаимодействие отсутствует.

Два гидрата, розовые орторомбические кристаллы $\left[\mathrm{Co}\left(\mathrm{H}_{2} \mathrm{O}\right)_{2}(\mathrm{Hba})_{2}\right]_{n}(\mathbf{1 0})$ и красные моноклинные кристаллы $\left[\mathrm{Co}\left(\mathrm{H}_{2} \mathrm{O}\right)_{4}(\mathrm{Hba})_{2}\right](\mathbf{1 1})$, кристаллизуются из водного раствора при $90{ }^{\circ} \mathrm{C}$ и комнатной температуре соответственно [12]. Коричневые кристаллы безводного [Co(Hba) $)_{n}$ получены нагреванием кристаллов 10 и 11 при $260^{\circ} \mathrm{C}$ в течение 15 мин. Все комплексы октаэдрические и связаны с лигандами только через атомы кислорода (рис. 7, таблица). Таким образом, 


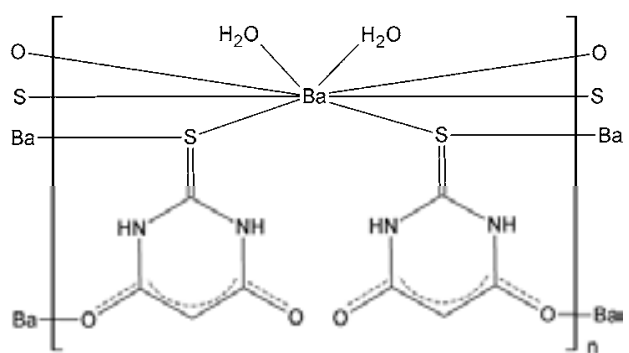

a)

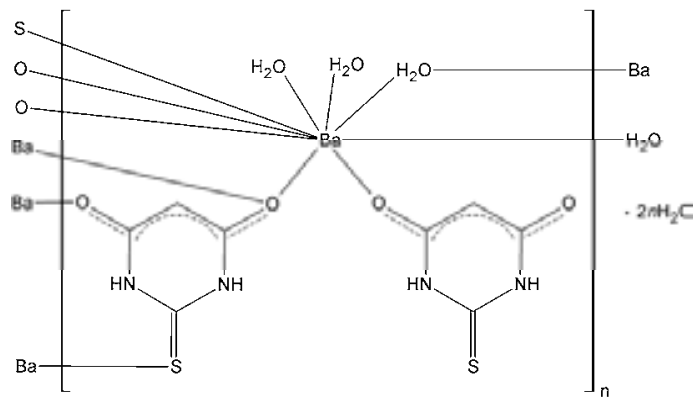

b)

Рис. 6. Строение комплексов $8(a)$ и $9(b)$

Fig. 6. Structure of complexes $\mathbf{8}$ (a) and $\mathbf{9}$ (b)

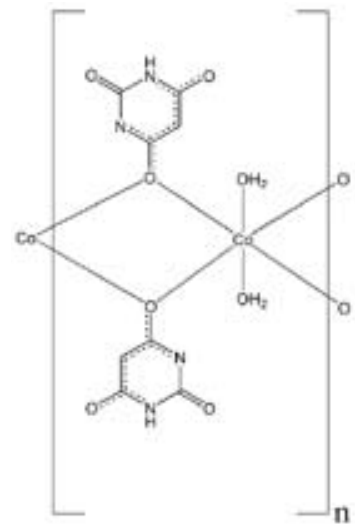

a)

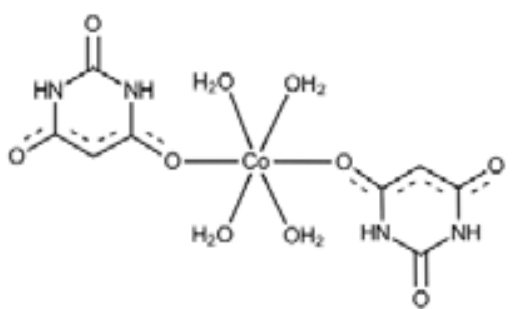

b)

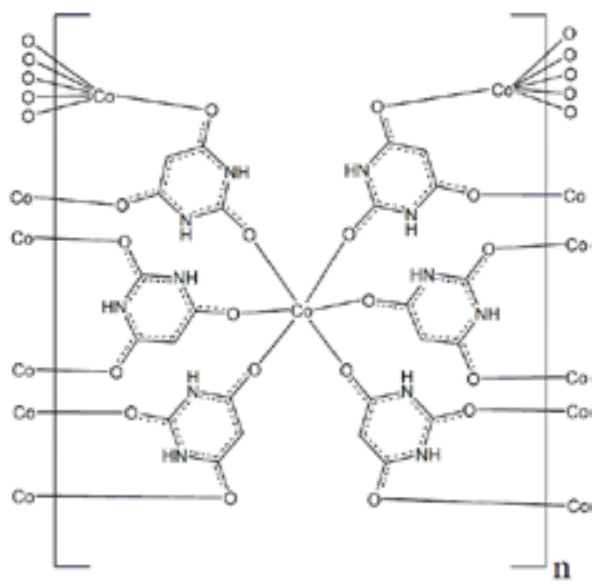

c)

Рис. 7. Координационное окружение $\mathrm{Co}(\mathrm{II})$ в $10(a), \mathbf{1 1}(b)$ и $\left[\mathrm{Co}(\mathrm{Hba})_{2}\right]_{n}(c)$

Fig. 7. The coordination environment of $\mathrm{Co}(\mathrm{II})$ in $\mathbf{1 0}(a), \mathbf{1 1}(b)$, and $\left[\mathrm{Co}(\mathrm{Hba})_{2}\right]_{n}(c)$ 
повышение температуры кристаллизации приводит к образованию барбитурата кобальта(II) с меньшим количеством координированных молекул воды. При нагревании этих комплексов при $260^{\circ} \mathrm{C}$ образуется безводный комплекс.

Симметрия кристаллов гидратов 10,11 и безводного комплекса зависит от числа гидратированных молекул воды. Соединение 11 имеет островную структуру. Два О-координированных барбитурат-иона, находящиеся в транс-положении, и четыре концевые молекулы воды образуют октаэдрическое окружение центрального иона Со(II). В комплексе 10 барбитурат-ион действует как О,О-мостиковый лиганд. Пары мостиковых ионов $\mathrm{Hba}^{-}$связывают два соседних иона Со(II) с образованием бесконечной 1D линейной цепи. Ближайшее расстояние Со‥Со в линейной цепи комплекса 10 равно 3.280(3) А̊. Структуры 10 и 11 стабилизированы водородными связями и $\pi-\pi$-взаимодействием. В безводном $\left[\mathrm{Co}(\mathrm{Hba})_{2}\right]_{n}$ каждый $\mu_{3}$-мостиковый ион $\mathrm{Hba}^{-}$связан с тремя ионами Сo(II) с образованием трехмерной структуры $3 \mathrm{D}$. В отличие от 10 и 11 в $\left[\mathrm{Co}(\mathrm{Hba})_{2}\right]_{n}$ в координации органического лиганда принимают участие все три атома кислорода. Снижение количества молекул воды в соединениях приводит к изменению способа координации лиганда от концевого до $\mu_{2}-\mathrm{O}, \mathrm{O}$ и далее до $\mu_{3}-\mathrm{O}, \mathrm{O}$, $\mathrm{O}^{\prime \prime}$ с структуры вещества: комплекс 11 имеет островную, комплекс 10 - цепочечную и $\left[\mathrm{Co}(\mathrm{Hba})_{2}\right]_{n}$ - каркасную структуру. При нагревании происходит последовательная дегидратация 11:

$$
\left[\mathrm{Co}\left(\mathrm{H}_{2} \mathrm{O}\right)_{4}(\mathrm{Hba})_{2}\right] \rightarrow\left[\mathrm{Co}\left(\mathrm{H}_{2} \mathrm{O}\right)_{2}(\mathrm{Hba})_{2}\right]_{n} \rightarrow\left[\mathrm{Co}(\mathrm{Hba})_{2}\right]_{n} .
$$

Синтезированы три гидрата $\left[\mathrm{Mn}\left(\mathrm{H}_{2} \mathrm{O}\right)_{x}(\text { Detba })_{2}\right](x=2,3.33$ или 4) [13], имеющие разное строение. Соединения кристаллизовали из водного раствора, полученного при взаимодействии нейтрализованного $\mathrm{NaOH}$ водного раствора HDetba c $\mathrm{MnCl}_{2} \cdot 4 \mathrm{H}_{2} \mathrm{O}$. Почти бесцветные кристаллы полимера $\left[\mathrm{Mn}\left(\mathrm{H}_{2} \mathrm{O}\right)_{2}(\text { Detba })_{2}\right]_{n}(\mathbf{1 2})$ осаждались в результате быстрого охлаждения вязкого концентрированного раствора до минус $3{ }^{\circ} \mathrm{C}$. Розовый трехъядерный комплекс $\left[\mathrm{Mn}_{3}\left(\mathrm{H}_{2} \mathrm{O}\right)_{10}(\mathrm{Detba})_{6}\right](\mathbf{1 3})$ выделен при медленном испарении воды из концентрированного раствора (как в синтезе 12) при комнатной температуре в течение 6 месяцев. Бледно-розовые кристаллы $\left[\mathrm{Mn}\left(\mathrm{H}_{2} \mathrm{O}\right)_{4}(\text { Detba) })_{2}\right] \cdot \mathrm{H}_{2} \mathrm{O}(\mathbf{1 4})$ также получены из концентрированного водного раствора, но при испарении растворителя на воздухе (в открытом стакане) при комнатной температуре.

В воде бесцветные кристаллы $\left[\mathrm{Mn}\left(\mathrm{H}_{2} \mathrm{O}\right)_{2}(\text { Detba })_{2}\right]_{n}$ в течение двух недель переходили в бледно-розовые кристаллы $\left[\mathrm{Mn}\left(\mathrm{H}_{2} \mathrm{O}\right)_{4}(\text { Detba })_{2}\right] \cdot \mathrm{H}_{2} \mathrm{O}$. Это позволяет рассматривать твердое соединение 14 как более термодинамически устойчивое в водном растворе по сравнению с 12. Следует отметить, что сами твердые кристаллы $\left[\mathrm{Mn}\left(\mathrm{H}_{2} \mathrm{O}\right)_{2}(\text { Detba })_{2}\right]_{n}$ устойчивы при комнатной температуре, по крайней мере, в течение 3 недель.

В комплексах 12-14 ион $\mathrm{Mn}(\mathrm{II})$ имеет октаэдрическое окружение и связан только с атомами кислорода. В 12 он связан с четырьмя $\mu_{2}$-мостиковыми ионами Detba ${ }^{-}$и двумя концевыми молекулами воды (рис. $8 a$ ). Молекулы воды расположены в транс-положении относительно друг друга, а ионы Detba- лежат в экваториальной плоскости полиэдра $\mathrm{MnO}_{6}$. Каждый ион Detba-

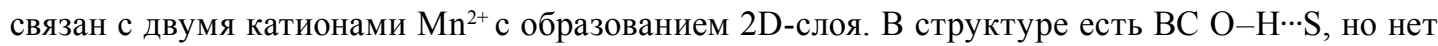
$\pi-\pi$-взаимодействия между ионами Detba ${ }^{-}$.

B структуре $\mathrm{Mn}_{3}\left(\mathrm{H}_{2} \mathrm{O}\right)_{10}(\text { Detba) })_{6}(\mathbf{1 3})$ содержится два независимых иона $\mathrm{Mn}^{2+}$, пять молекул воды и три иона Detba- К иону Mn1 координированы три концевых молекул воды, два концевых и один $\mu_{2}$-мостиковый ионы $\operatorname{Detba}^{-}$(рис. $8 b$ ). Октаэдрическое окружение Mn2 создает- 


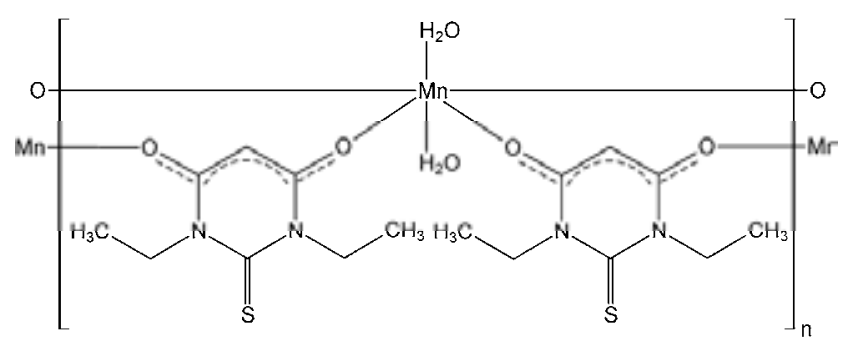

a)

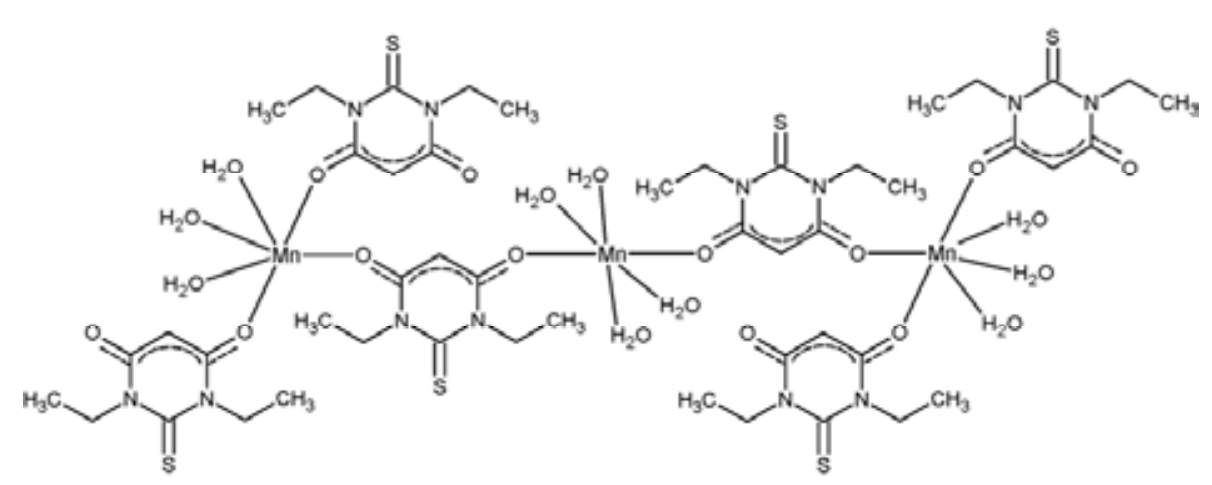

b)

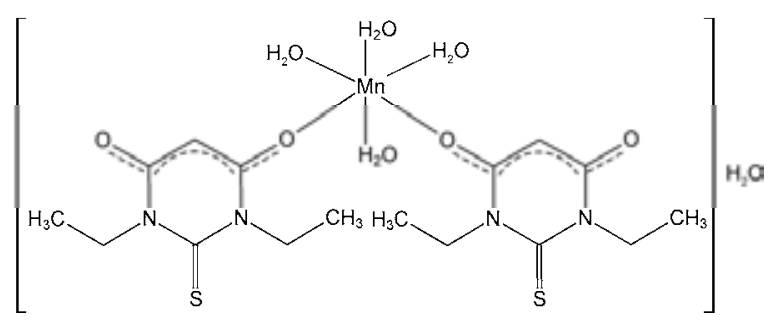

c)

Рис. 8. Строение $\left[\mathrm{Mn}\left(\mathrm{H}_{2} \mathrm{O}\right)_{2}(\text { Detba })_{2}\right](a),\left[\mathrm{Mn}_{3}\left(\mathrm{H}_{2} \mathrm{O}\right)_{10}(\text { Detba })_{6}\right](b)$ и $\left[\mathrm{Mn}\left(\mathrm{H}_{2} \mathrm{O}\right)_{4}(\text { Detba })_{2}\right] \cdot \mathrm{H}_{2} \mathrm{O}(c)$

Fig. 8. Structure of $\left[\mathrm{Mn}\left(\mathrm{H}_{2} \mathrm{O}\right)_{2}(\text { Detba })_{2}\right](a),\left[\mathrm{Mn}_{3}\left(\mathrm{H}_{2} \mathrm{O}\right)_{10}(\text { Detba })_{6}\right](b)$ and $\left[\mathrm{Mn}\left(\mathrm{H}_{2} \mathrm{O}\right)_{4}(\text { Detba })_{2}\right] \cdot \mathrm{H}_{2} \mathrm{O}(c)$

ся двумя $\mu_{2}$-мостиковыми ионами Detba- и четырьмя молекулами $\mathrm{H}_{2} \mathrm{O}$. Два независимых иона Detba ${ }^{-}$являются концевыми, а один - $\mu_{2}$-мостиковым. Межмолекулярные водородные связи $\mathrm{O}-\mathrm{H} \cdots \mathrm{O}, \mathrm{O}-\mathrm{H} \cdots \mathrm{S}$ и $\pi-\pi$-взаимодействие («голова-к-хвосту») стабилизируют структуру.

В комплексе 14 островного строения Mn(II) связан с двумя концевыми ионами Detba- и четырьмя концевыми молекулами $\mathrm{H}_{2} \mathrm{O}$ (рис. 8 c). Ионы Detba- расположены в транс-положении. Водородные связи $\mathrm{O}-\mathrm{H} \cdots \mathrm{O}, \mathrm{O}-\mathrm{H} \cdots \mathrm{S}$ образуют трехмерную сеть. $\pi-\pi$-взаимодействие между ионами Detba- отсутствует.

Как следует из рассмотренного выше материала, наибольшее число гидратов нейтральных барбитуратных комплексов получено в случае $\left[\mathrm{Mn}\left(\mathrm{H}_{2} \mathrm{O}\right)_{x}(\text { Detba })_{2}\right](x=2,3.33$ или 4). Стехиометрические соотношения между гидратами 1,3-диэтил-2-тиобарбитурата $\mathrm{Mn}$ (II) таковы: 


$$
\begin{array}{cc}
-5 \mathrm{H}_{2} \mathrm{O} & -4 \mathrm{H}_{2} \mathrm{O} \\
3\left[\mathrm{Mn}\left(\mathrm{H}_{2} \mathrm{O}\right)_{4}(\text { Detba })_{2}\right] \cdot \mathrm{H}_{2} \mathrm{O}(\mathbf{1 4}) \rightarrow\left[\mathrm{Mn}_{3}\left(\mathrm{H}_{2} \mathrm{O}\right)_{10}(\text { Detba })_{6}\right](\mathbf{1 3}) \rightarrow 3\left[\mathrm{Mn}\left(\mathrm{H}_{2} \mathrm{O}\right)_{2}(\text { Detba })_{2}\right](\mathbf{1 2}) .
\end{array}
$$

Систематический поиск (скрининг) различных гидратов нейтральных барбитуратных комплексов пока специально не проводился. В рассмотренных выше работах [7-13] всего лишь установлено, что при изменении условий кристаллизации из водного раствора осаждаются соединения разного состава и строения внутренней координационной сферы. Причем это наблюдается для ионов металлов разного электронного строения. Различие в молекулярной структуре гидратов отражается на супрамолекулярном уровне, в частности на системе ВС и $\pi-\pi$-взаимодействии барбитуратных лигандов.

В целом, при рассмотрении возможности получения кристаллических нейтральных гидратных комплексов следует принимать во внимание координирующие способности центральных ионов металлов, полифункциональных лигандов и молекул воды. В случае полифункциональных лигандов с потенциально разной дентатностью и/или мостиковым способом координации к ионам металлов всегда стоит ожидать образование нескольких нейтральных комплексов с разным количеством координированных молекул воды. Этому также благоприятствует способность молекул воды быть как концевыми, так и мостиковыми лигандами, например как в случае полимерных тиобарбитуратов натрия, стронция и бария (таблица, рис. 2 , 5 и 6). Кристаллизация разных гидратов также ожидается для комплексов ионов металлов, легко образующих разные координационные полиэдры, например щелочноземельных (таблица, рис. 4-6). Способность к координации нейтральных лигандов, в том числе и совместно с координацией анионов как в $\left[\mathrm{K}\left(\mathrm{H}_{2} \mathrm{O}\right)\left(\mathrm{H}_{2} \mathrm{tba}\right)(\mathrm{Htba})\right]_{n}$, расширяет список гидратных комплексов, которые можно выделить из водного раствора. Совокупность перечисленных возможностей позволяет предположить, что путем изменения условий кристаллизации для каждого из пока структурно охарактеризованных нейтральных комплексов металлов с полифункциональными лигандами можно получить несколько новых фаз с отличающимся количеством координированных молекул воды. Это существенно расширяет круг доступных соединений, которые могут найти практическое применение. Остается неясным, насколько широко это явление распространено для нейтральных комплексов металлов с другими полифункциональными лигандами. Условия образования и роста зародышей новых фаз в настоящее время трудно предсказуемы, так как образование активных центров кристаллизации часто происходит в результате лишь нескольких актов взаимодействия, а иногда - лишь одного [20, c. 509]. Это означает, что в отличие от большинства химических реакций, которые являются результатом взаимодействия $\approx 10^{20}$ частиц, кристаллизация не подчиняется статистическим закономерностям, присущим ансамблям большого числа частиц. Поэтому трудно обоснованно выбрать подходящие условия получения каждого из твердых гидратов. Например, все гидраты $\left[\mathrm{Mn}\left(\mathrm{H}_{2} \mathrm{O}\right)_{x}(\text { Detba })_{2}\right](x=2,3.33$ или 4) осаждаются практически из одного и того же маточного раствора при разнице температуры всего около $20^{\circ} \mathrm{C}$. Понижение температуры кристаллизации может приводить к осаждению как более обогащенного водой комплекса, например $\left[\mathrm{Co}\left(\mathrm{H}_{2} \mathrm{O}\right)_{4}(\mathrm{Hba})_{2}\right]$, так и менее обогащенного, в частности $\left[\mathrm{Mn}\left(\mathrm{H}_{2} \mathrm{O}\right)_{2}(\mathrm{Detba})_{2}\right]_{n}$ (таблица). Следует отметить, что вероятность успешного получения каждого твердого гидрата нейтрального комплекса также существенно зависит от способа упаковки и энергии кристаллической решетки, особенностей супрамолекулярных взаимодействий, кинетики образова-

$$
-412-
$$


ния кристаллов (и т.д.) и, конечно, затраченного исследователем времени на изучение кристаллизации при различных условиях.

Следует обратить внимание на то, что целостной системы классификации гидратов, учитывающей разное распределение молекул воды между внутренней координационной и внешней сферой комплекса, пока нет. В настоящей работе рассмотрены мономерные и полимерные комплексы металлов, состав которых отражается обобщенной стехиометрической формулой $\left[\mathrm{M}\left(\mathrm{H}_{2} \mathrm{O}\right)_{n-k} \mathrm{~L}_{\mathrm{m}}\right]^{0} \cdot p \mathrm{H}_{2} \mathrm{O}$. Нейтральный комплекс $\left[\mathrm{M}\left(\mathrm{H}_{2} \mathrm{O}\right)_{n} \mathrm{~L}_{\mathrm{m}}\right]^{0}$ и кристаллогидраты $\left[\mathrm{M}\left(\mathrm{H}_{2} \mathrm{O}\right)_{n-k} \mathrm{~L}_{\mathrm{m}}\right]^{0} k \mathrm{H}_{2} \mathrm{O}$ имеют один и тот же количественный состав, но отличаются пространственным строением и свойствами, т.е. по общепринятому определению они являются изомерами. Другие попытки учесть в классификации гидратов различное распределение молекул воды между внутренней и внешней координационными сферами, а также способ их координации нам неизвестны. Нейтральные соединения, не содержащие кристаллизационную воду $\left[\mathrm{M}\left(\mathrm{H}_{2} \mathrm{O}\right)_{n} \mathrm{~L}_{m}\right]^{0}$ и $\left[\mathrm{M}\left(\mathrm{H}_{2} \mathrm{O}\right)_{n-k} \mathrm{~L}_{m}\right]^{0}(\mathrm{k} \neq 0)$ и отличающиеся составом, а часто и формой координационных полиэдров, надо рассматривать как индивидуальные. Поэтому все рассмотренные соединения (таблица), кроме 6 и 7, являются разными. Комплекс $\left.\left[\mathrm{M}_{(\mathrm{H}} \mathrm{O}\right)_{n} \mathrm{~L}_{m}\right]^{0}$ и каждый из кристаллогидратов $\left[\mathrm{M}\left(\mathrm{H}_{2} \mathrm{O}\right)_{n-k} \mathrm{~L}_{m}\right]^{0} \cdot p \mathrm{H}_{2} \mathrm{O}(k \neq 0$ и $k \neq p)$ также надо рассматривать как индивидуальные соединения. Если $k=0$ и $p>0$, то соединения $\left[\mathrm{M}\left(\mathrm{H}_{2} \mathrm{O}\right)_{n} \mathrm{~L}_{m}\right]^{0} \cdot p \mathrm{H}_{2} \mathrm{O}$ логично считать кристаллогидратами одного и того же соединения $\left[\mathrm{M}\left(\mathrm{H}_{2} \mathrm{O}\right)_{n} \mathrm{~L}_{m}\right]^{0}$. Тогда комплекс $\left[\mathrm{Sr}\left(\mathrm{H}_{2} \mathrm{O}\right)_{4}(\mathrm{Htba})_{2}\right]_{n} \cdot n \mathrm{H}_{2} \mathrm{O}$ (7) является кристаллогидратом $\left[\mathrm{Sr}_{2}\left(\mathrm{H}_{2} \mathrm{O}\right)_{4}(\mathrm{Htba})_{2}\right]_{n}(\mathbf{6})$, хотя строение координационного полиэдра $\mathrm{Sr}(\mathrm{II})$ и способы координации лиганда $\mathrm{Htba}^{-}$в этих комплексах разные (рис. 5, таблица).

\section{Заключение}

Установлено, что для каждого иона металла, независимо от его природы, при изменении условий кристаллизации из водного раствора осаждаются нейтральные барбитуратные комплексы разного состава и строения, содержащие различные количества координированных молекул воды. Они отличаются супрамолекулярной организацией, в частности системой ВС и $\pi-\pi$-взаимодействием между барбитуратными лигандами.

В общем случае для полифункциональных лигандов с потенциально разной дентатностью и/или мостиковым способом координации к ионам металлов всегда следует ожидать образование нейтральных комплексов с разным количеством координированных молекул воды. Совместная координация анионной и нейтральных форм одного и того же лиганда расширяет список гидратных комплексов, которые можно выделить из водного раствора. Кроме того, кристаллизация нескольких гидратов также ожидается для ионов металлов, легко образующих разные координационные полиэдры. Можно предположить, что для каждого из пока структурно охарактеризованных нейтральных комплексов металлов с полифункциональными лигандами можно получить несколько новых фаз с отличающимся количеством координированных молекул воды, что расширяет круг доступных соединений.

Работа выполнена в рамках государственного задания Минобрнауки РФ Сибирскому федеральному университету в 2017-2019 г2. (4.7666.2017/БЧ). 


\section{Список литературы}

1. Braga D., Grepioni F., Desiraju G.R. Crystal Engineering and Organometallic Architecture. Chemical Reviews 1998. Vol. 98(4), P. 1375-1405.

2. Goodman L.S., Gilman A. Pharmacological Basis of Therapeutics. London: The MacMillan Company, 1970. $1856 \mathrm{p}$.

3. Masoud M.S., Khalil E.A., Hindawy A.M., Ali E.A., Mohamed E.F. Spectroscopic studies on some azo compounds and their cobalt, copper and nickel complexes. Spectrochimica Acta 2004. Vol. 60, P. 2807-2817.

4. Mahmudov K.T., Kopylovich M.N., Maharramov A.M., Kurbanova M.M., Gurbanov A.V., Pombeiro A.J. Barbituric acids as a useful tool for the construction of coordination and supramolecular compounds. Coordination Chemistry Reviews 2014. Vol. 265, P. 1-37.

5. Головнев Н.Н., Молокеев М.С. 2-тиобарбитуровая кислота и ее комплексы с металлами: синтез, структура и свойства. Красноярск: Сиб. федер. ун-т, 2014. 252 с. [Golovnev N.N., Molokeev M.S. 2-Thiobarbituric acid and its complexes with metals: synthesis, structure and properties. Krasnoyarsk: Siberian Federal University, 2014. 252 p. (In Russ.)]

6. Cambridge Structural Database, Univ. of Cambridge, Cambridge, UK, 2017.

7. Golovnev N.N., Molokeev M.S., Sterkhova I.V., Atuchin V.V., Sidorenko M.Y. Hydrated pseudopolymorphs $\left[\mathrm{Na}_{2}\left(\mathrm{H}_{2} \mathrm{O}\right)_{\mathrm{x}}\right](2 \text {-thiobarbiturate })_{2}(\mathrm{x}=3,4,5)$ : crystal structure, spectroscopic and thermal properties. Journal of Coordination Chemistry 2016. Vol. 69(21), P. 3219-3230.

8. Golovnev N.N., Molokeev M.S. Bridging behaviour of the 2-thiobarbiturate anion in its complexes with $\mathrm{Li}(\mathrm{I})$ and $\mathrm{Na}(\mathrm{I})$. Acta Crystallographica Section C: Crystal Structure Communications 2013. Vol. 69(7), P. 704-708.

9. Golovnev N.N., Molokeev M.S., Vereshchagin S.N., Atuchin V.V. Calcium and strontium thiobarbiturates with discrete and polymeric structures. Journal of Coordination Chemistry 2013. Vol. 66(23), P. 4119-4130.

10. Golovnev N.N., Molokeev M.S. Structures of bis (2-thiobarbiturato-O) tetraaquamagnesium and catena-[( $\mu 2-2$-thiobarbiturato-O, O)(2-thiobarbiturato-O) bis ( $\mu 2$-aqua) diaquastrontium] monohydrate. Russian Journal of Inorganic Chemistry 2014. Vol. 59(2), P. 72-78.

11. Golovnev N. N., Molokeev M.S. Crystal structures of two barium 2-thiobarbiturate complexes. Journal of Structural Chemistry 2014. Vol. 55(5), P. 871-878.

12. Golovnev N.N., Solovyov L.A., Lesnikov M.K., Vereshchagin S.N., Atuchin V.V. Hydrated and anhydrous cobalt (II) barbiturates: Crystal structures, spectroscopic and thermal properties. Inorganica Chimica Acta 2017. Vol. 467, P. 39-45.

13. Golovnev N.N., Molokeev M.S., Sterkhova I.V., Lesnikov M.K., Atuchin V.V. Coordination effects in hydrated manganese(II) 1,3-diethyl-2-thiobarbiturates and their thermal stability. Polyhedron 2017. Vol. 134, P. 120-125.

14. Clarke H.D., Arora K.K., Bass H., Kavuru P., Ong T.T., Pujari T., Wojtas L., Zaworotko M.J. Structure-stability relationships in cocrystal hydrates: does the promiscuity of water make crystalline hydrates the Nemesis of crystal enlineering? Crystal Growth \& Design 2010. Vol. 10, P. 2152-2167.

15. Li B., Li W., Ye L., Hou G.F., Wu L.X. Poly[di- $\mu_{2}$-aqua- $\mu_{4}$-chlorido- $\mu_{4}-(2-$ mercaptopyrimidine4,6-diolato- $\left.\kappa^{4} \mathrm{O}: \mathrm{O}: \mathrm{O}^{\prime}: \mathrm{O}^{\prime}\right)$-disodium(I)]. Acta Crystallographica Section E 2010. Vol. 66, P. 15461546 . 
16. Kubicki M., Owczarzak A., Balas V.I., Hadjikakou S.K. Structural characterization of polymeric potassium salts with 2-thiobarbituric acid: influence of organotin(IV) chlorides on potassium cation solvation. Journal of Coordination Chemistry 2012. Vol. 65(7), P. 1107-1117.

17. Balas V.I., Verginadis I.I., Geromichalos G.D. et al. Synthesis, structural characterization and biological studies of the triphenyltin(IV) complex with 2-thiobarbituric acid. European Journal of Medicinal Chemistry 2011. Vol. 46(7), P. 2835-2844.

18. Chierotti M.R., Gaglioti K., Gobetto R., Braga D., Grepioni F., Maini L. From molecular crystals to salt co-crystals of barbituric acid via the carbonate ion and an improvement of the solid state properties. CrystEngComm 2013. Vol. 15, P. 7598-7605.

19. Gryl M., Stadnicka K. Rubidium 2,4,6-trioxo-1,3-diazinan-5-ide-1,3-diazinane -2,4,6-trione-water (1/1/1). Acta Crystallographica Section E 2011. Vol. 67, P. 571-572.

20. Стид Дж. В., Этвуд Дж. Л. Супрамолекулярная химия. М.: Академкнига, 2007. 480 с. [Steed J.W., Atwood J.L. Supramolecular Chemistry. Moscow: IKTs Akademkniga, 2007. 480 p. (In Russ.)] 